\title{
Search for space charge effects in the ICARUS T600 LAr-TPC
}

\author{
Marta Torti ${ }^{1,2, a}$ on behalf of the ICARUS Collaboration \\ ${ }^{1}$ Department of Physics, University of Pavia, Italy \\ ${ }^{2}$ Istituto Nazionale di Fisica Nucleare (INFN), Section of Pavia, Italy
}

\begin{abstract}
Space charge in Liquid Argon Time Projection Chamber is due to the accumulation of positive ions, produced by ionizing tracks crossing the detector, which slowly flow toward the cathode. As a consequence, electric field distortions may arise, thus hindering the possibility to produce faithful 3D images of the ionizing events. The presence of space charge becomes relevant for large TPCs operating at surface or at shallow depths, where cosmic ray flux is high. These effects could interest the next phase of the ICARUS T600 detector, which will be deployed at shallow depths as a Far Detector for Short Baseline Neutrino experiment at FNAL dedicated to sterile neutrino searches. In 2001, the first ICARUS T600 module (T300) operated at surface in Pavia (Italy), recording cosmic ray data. In this work, a sample of cosmic muon tracks from the 2001 run was analyzed and results on space charge effects in LAr-TPCs are shown.
\end{abstract}

\section{Introduction}

ICARUS T600 is a Liquid Argon Time Projection Chamber (LAr-TPC) that took data from 2010 until 2013 at the Gran Sasso Laboratory (LNGS) in underground conditions [1]. The next phase of the detector foresees its deployment as a Far Detector for Short Baseline Neutrino (SBN) experiment at Fermi National Accelerator Laboratory (FNAL) in USA, dedicated to sterile neutrino searches [2]. This new program will involve three LAr-TPCs, placed at different positions from a neutrino source, the Booster Neutrino Beam (BNB): in addition to the T600 $(\sim 600 \mathrm{~m})$, there will be MicroBoone (now running), placed at $\sim 470 \mathrm{~m}$, and SBND, at $\sim 110 \mathrm{~m}$. The goal of the SBN program is to give a $5 \sigma$ answer to the LSND anomaly [3], that can be explained with the presence of a fourth sterile neutrino state.

At FNAL the detector will not operate in underground environment, but it will be at shallow depths, with a high cosmic ray flux. In this condition, space charge effects could be relevant. Due to the accumulation in the drift region of positive ions $\left(\mathrm{Ar}^{+}\right)$, produced by ionizing tracks crossing the detector, electric field distortions may arise. In fact, ions survive in the drift region for a very long time because the positive ion mobility in LAr, $\mu_{i} \sim 1.6 \cdot 10^{-3} \mathrm{~cm}^{2} \mathrm{~s}^{-1} V^{-1}$, is much smaller than the free electrons one, $\mu_{e} \sim 500 \mathrm{~cm}^{2} \mathrm{~s}^{-1} V^{-1}$.

An uniform electric field is fundamental to product faithful 3D images of the ionizing events, in order to have an uniform drift velocity and so the proportionality between drift time and drift coordinate.

ae-mail: marta.torti@pv.infn.it 


\section{The ICARUS T600 detector}

ICARUS (Image Cosmic And Rare Underground Signal) T600 detector [4] consists of a large cryostat split into two identical, adjacent half-modules $\left(3.6 \times 3.9 \times 19.6 \mathrm{~m}^{3}\right.$ each $)$ and filled with about 760 tons of ultra-pure liquid argon: each half-module houses two TPCs (left and right chambers) separated by a common cathode. A uniform electric field $\left(E_{D}=500 \mathrm{~V} / \mathrm{cm}\right)$ is applied to the LAr bulk.

Charged particles, crossing LAr, produce ionization along their path. Thanks to the low transverse diffusion of charge in LAr, the images of the tracks, produced by ionization electron clouds, are preserved and, drifting along the electric field lines $\left(v_{\text {drift }} \sim 1.6 \mathrm{~mm} / \mu \mathrm{s}\right)$, are projected onto the anode, as illustrated in figure 1.

The TPC anode is made of three parallel planes of wires, $3 \mathrm{~mm}$ apart, facing the $1.5 \mathrm{~m}$ drift path $\left(\sim 1 \mathrm{~ms}\right.$ drift time). Wires are oriented on each plane at a different angle $\left(0^{\circ}, \pm 60^{\circ}\right)$ with respect to the horizontal direction. Therefore, combining the wire/drift coordinates on each plane at a given drift time, a three-dimensional reconstruction of the ionizing event can be obtained, with a resolution of about $1 \mathrm{~mm}^{3}$. The drift coordinate is obtained by the measurement of the absolute time of the ionizing event, with the scintillation light detection, combined with the electron drift velocity information.

Positive ions are drifted toward the cathode too, but with a velocity $\sim 2000$ times smaller than the free electrons one, corresponding to a drift time of the order of seconds. If events rate is too high, ions, while reaching the cathode, can interact with ionizing electrons from other events, perturbing their drift path.

A first physical run, with a single T300 module, was performed in 2001 in Pavia (Italy), collecting cosmic ray-induced events. The T600 detector successfully operated from 2010 to 2012 in Gran Sasso underground laboratories (LNGS), where it took data from the CNGS (CERN Neutrino to Gran Sasso) neutrino beam and with cosmic rays, to study neutrino oscillations. After the shut down of the beam, it collected data, focusing on cosmic rays, up to June 2013, when the phase of decommissioning began.

\section{Data sample and method}

To study possible space charge effects in the T600 detector, a sample of cosmic muon bundles has been considered. These data were taken during the test run in Pavia (Italy), in 2001, where only a T300 module operated at surface. Muon bundles have been used because they are composed of high energy parallel muons crossing entirely the drift region.

The whole sample included 129 muons. Each track was selected with a visual scanning procedure and delta rays were removed. First and last $1.5 \mathrm{~cm}$ of each track were not reconstructed, to avoid possible boundary dis-uniformities due to the presence of the wires and cathode planes.

Muon tracks observed to bend, following a parabola, could suggest the presence of possible positive ion accumulation: in fact, due to the dependence of the electron drift velocity on the electric field, the ionization electron arrival time $T_{s c}$ on the anode in presence of space charge effects (apparent track in the figure 2), will be delayed with respect to the time $T_{u}$ observed in the case of uniform electric field (ionizing track in figure 2). Thus, the bending parameter $\Delta T=T_{s c}-T_{u}$ is evaluated to search for possible delays.

For each muon track, $T_{s c}$ is measured wire by wire, while $T_{u}$ is evaluated sketching the muon as a straight line fitting the starting $5 \mathrm{~cm}$ and the last $5 \mathrm{~cm}$ of the track, where the electric field is fixed. Then, $\Delta T$ is evaluated for every point of the remaining part of the track.

\section{Results and comparison with simulation}

As a first attempt to identify the possible bending of the tracks, for each centimeter of the drift path, the average $\Delta T$ from all tracks was calculated; the mean value of the distribution was found to be 


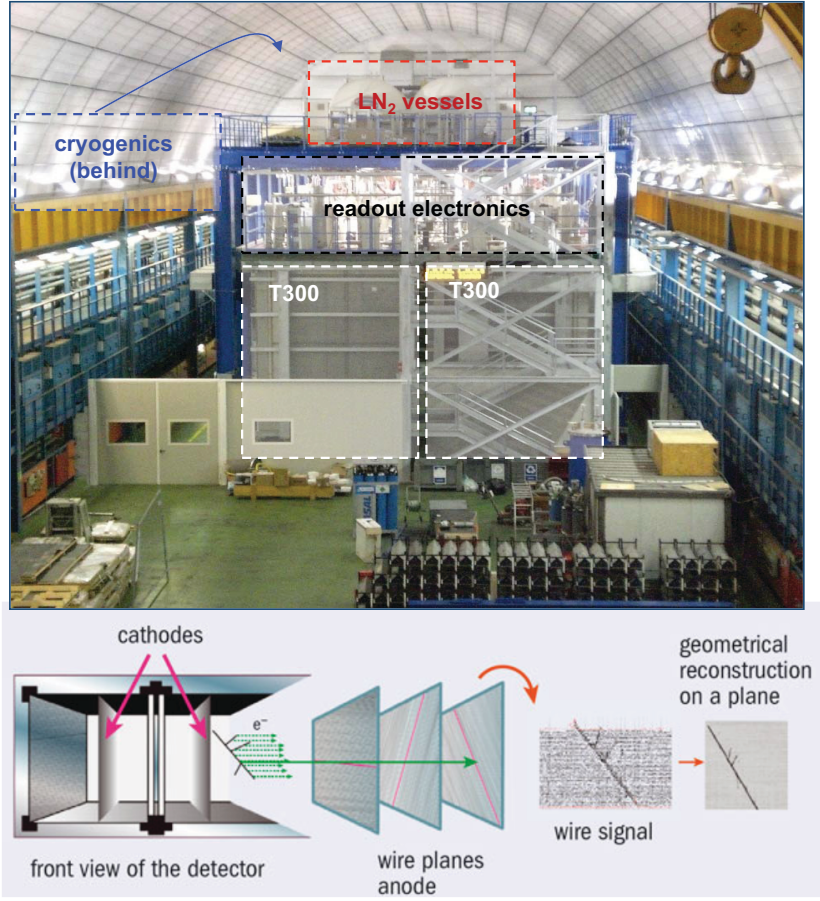

Figure 1. Top: ICARUS T600 detector in the Hall B of LNGS, with a schematic view of different parts. The two T300 modules are enlightened, such as the space occupied by the readout electronics and the cryogenics. Bottom: TPC working principle: when a charged particle cross the detector, electrons are drifted through the wire planes, where the charge is collected by the three wire planes. By appropriate voltage biasing, the first two , Induction1 and Induction2 planes, provide signals in a non-destructive way; finally the ionization charge is collected and measured on the last one, the Collection plane. Combining all the information, it is possible to have a $3 \mathrm{D}$ reconstruction of the track.

$\Delta T_{\text {mean }}=0.18 \mathrm{~cm}$, with a RMS of $0.67 \mathrm{~cm}$. Then, $\Delta T$ as a function of the drift coordinate was compared with a numerical simulation (figure 3), in which possible electric field distortions caused by space charge were taken into account, due to high cosmic ray flux at surface. Data are in good agreement with the simulation, apart from the first and last sections, due to the $\Delta T$ calculation method. The maximum deviation from the linear fit of the tracks was found to be $\Delta T_{\max }=0.34 \mathrm{~cm}$.

The easiest way to link these results to space charge effects is to analyze cosmic ray data collected underground at LNGS, where the cosmic ray flux is much smaller then at surface and these effects should not be present. A sample of 76 muon tracks was then analyzed as described above. As expected, in LNGS sample, the result is compatible with the absence of these effects: no deviation from the straight track was observed along the entire drift path (figure 4). The mean value of the global $\Delta T$ distribution for this sample is $\Delta T_{\text {mean }}=0.04 \mathrm{~cm}$ with a RMS of $0.37 \mathrm{~cm}$.

To better understand if the curvature measured in the Pavia surface sample was due to space charge or to other effects, the analysis was repeated by splitting data between the left (69 muons) and the right (60 muons) TPC chambers of the T300 module. The two chambers show a different distortion behavior (figure 5): in the right chamber the space charge effects were marginal, while in the left one the bending was more accentuated. This can not be explained in terms of space charge: different 

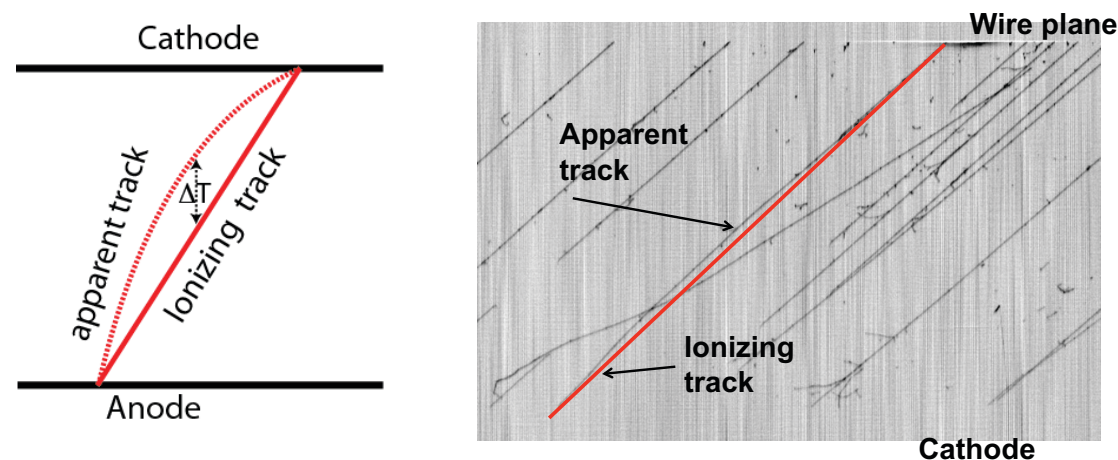

Figure 2. Left: schematic view of the bending parameter calculation. Right: example of bending parameter calculation on real data.

thermal insulation and LAr re-circulation conditions during the 2001 Pavia run could be responsible of the differences between the chambers.

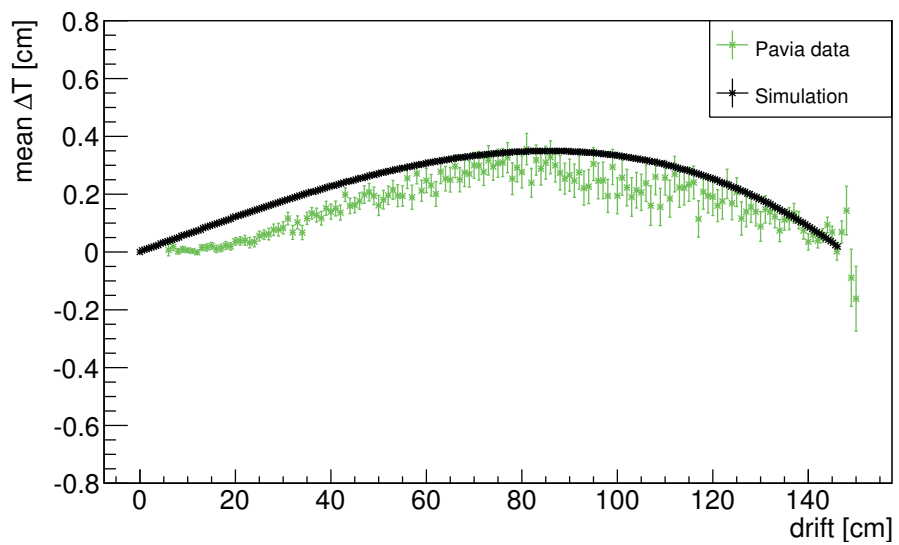

Figure 3. Comparison between Pavia data sample results and simulation of space charge effects due to high cosmic ray flux at surface.

\section{Conclusions}

Results here presented suggest the presence of small space charge effects inside the ICARUS T300 detector operated at surface in Pavia 2001 run, with a maximum track bending $\Delta T_{\max } \sim 0.3 \mathrm{~mm}$. The analysis of a cosmic muon sample, collected underground at LNGS where this effect should not be present, produced a $\Delta T$ flat distribution, confirming that the track distortion observed at surface could be indeed due to space charge effects.

On the other hand, by analyzing the left and the right T300 chambers separately, the bending clearly 


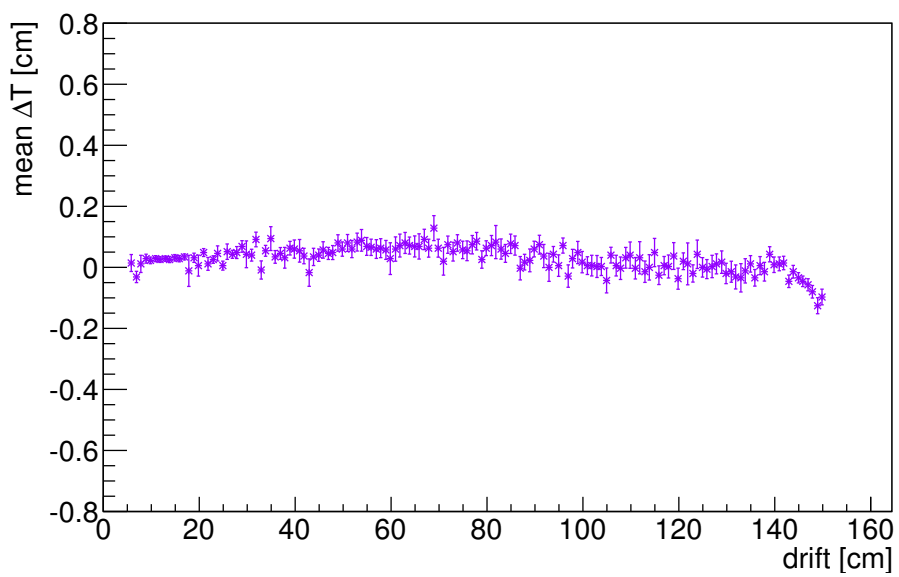

Figure 4. Results for LNGS data sample, collected at underground condition.

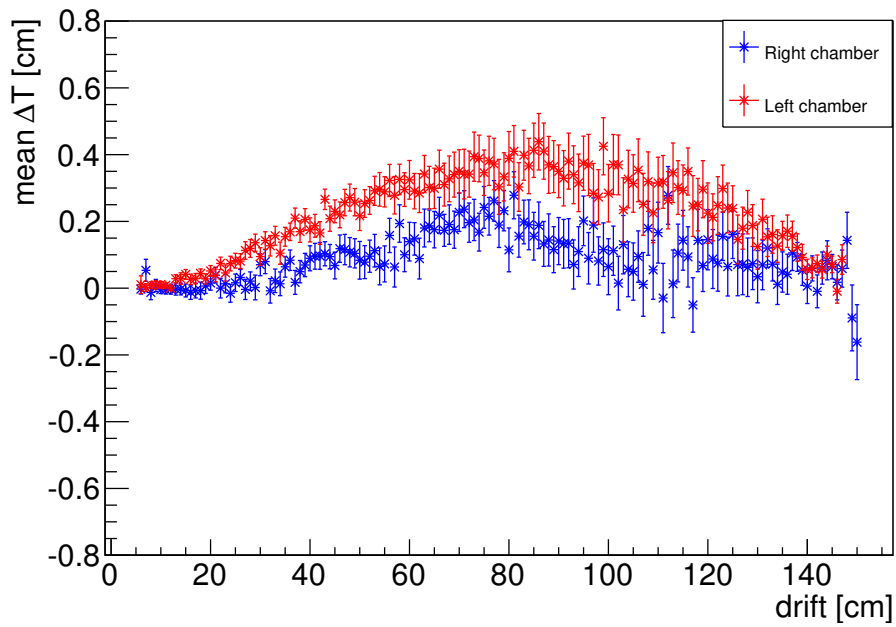

Figure 5. Results for Pavia data sample split between left and right TPC chamber of T300 module.

appears only in the left chamber: this behavior can not be directly ascribed to space charge effects, and more studies are required to fully understand the origin of this small surface muon track bending.

\section{References}

[1] C. Rubbia et al., JINST 6 (2011) P07011.

[2] R. Acciari et al., A proposal for a three detector Short Baseline Neutrino oscillation program in the Fermilab Booster Neutrino Beam, arXiv:1503.01520 
[3] A. Agiular et al. (LSND Collaboration), Phys. Rev. D, 64, 112007, 2001

[4] S. Amoruso et al.,Nucl. Inst. Meth., A527 , 329-410, 2004 